\title{
Effects of state anxiety on performance using a task-switching paradigm: An investigation of attentional control theory
}

\author{
NaZanin Derakshan \\ Birkbeck, University of London, London, England \\ SinÉAD SMYTH \\ University of Ulster, Coleraine, Northern Ireland \\ AND \\ Michael W. EySENCK \\ Royal Holloway, University of London, London, England
}

\begin{abstract}
Low- and high-anxious participants performed arithmetical tasks under task-switching or nontask-switching conditions. These tasks were low or high in complexity. The task on each trial was either explicitly cued or not cued. We assumed that demands on attentional control would be greater in the task-switching condition than in the nontask-switching condition, and would be greater with high-complexity tasks than with low-complexity ones. We also assumed that demands on attentional control would be greater when cues were absent rather than present. According to attentional control theory (Eysenck, Derakshan, Santos, \& Calvo, 2007), anxiety impairs attentional control processes required to shift attention optimally within and between tasks. We predicted that there would be greater negative effects of high state anxiety in the task-switching condition than in the nontask-switching condition. Our theoretical predictions were supported, suggesting that state anxiety reduces attentional control.
\end{abstract}

The processing efficiency theory (PET; Eysenck \& Calvo, 1992) was developed to explain the frequent adverse effects of anxiety on the performance of complex cognitive tasks. According to PET, high levels of state anxiety reduce the efficiency of cognitive processing and often lead to impaired performance. Specifically, anxiety affects the functioning of the working memory system which, according to Baddeley's (1986) original conception, consisted of the central executive (a limited capacity, attention-like system), the phonological loop (used for verbal rehearsal), and the visuospatial sketchpad (used for processing visual and spatial information). According to PET, the component of working memory most affected by anxiety is the central executive.

Support for PET comes from research showing that anxiety impairs performance when two concurrent tasks both use the central executive. Eysenck, Payne, and Derakshan (2005) conducted a systematic investigation of the effects of anxiety on the different components of working memory using the Corsi Blocks Test. This task was performed concurrently with different secondary tasks, each utilizing a different component of working memory. Adverse effects of anxiety on the Corsi task were obtained only when the secondary task required use of the central executive, suggesting that anxiety reduces its available capacity.

In spite of much empirical support for PET, Eysenck, Derakshan, Santos, and Calvo (2007) highlighted various limitations in that theory. Importantly, PET failed to specify the executive function(s) affected by anxiety. There is ongoing debate concerning the number and nature of such functions. However, in a systematic investigation, Miyake et al. (2000) used latent variable analysis to identify three major control functions of the central executive: inhibition, shifting, and updating. The inhibition function often involves using attentional control in a negative way to prevent attentional resources being allocated to taskirrelevant stimuli and responses (Friedman \& Miyake, 2004). The shifting function involves using attentional control in a positive way to shift the allocation of attention to maintain focus on task-relevant stimuli. According to attentional control theory (ACT; Eysenck et al., 2007), anxiety impairs the efficiency of the inhibition and shifting functions. There is direct support for the prediction that anxiety impairs the inhibition function (Derakshan, Ansari, Hansard, Shoker, \& Eysenck, 2009).

Miyake et al. (2000) reported that performance on taskswitching paradigms loads highly on the shifting func-

N. Derakshan, n.derakhshan@bbk.ac.uk 
tion. Task-switching paradigms involve comparing performance on blocks of trials involving one task (A or B) with performance on blocks of trials with $50 \%$ of trials involving Task A and 50\% Task B. Participants exposed to a task-switching procedure generally demonstrate a switching cost (Monsell, 2003). There is hardly any published research on the effects of anxiety on the shifting function. However, Ansari, Derakshan, and Richards (2008), using a mixed antisaccade task, found that highanxious individuals did not show the commonly found switch benefit that results from alternating between antiand prosaccade trials, suggesting that anxiety impaired the shifting function.

In order to test the effects of anxiety on task switching, we were motivated by the principles underlying the paradigm developed by Rubinstein, Meyer, and Evans (2001; Experiment 2) to form the basis for the present experiment. Rubinstein et al. presented mathematical problems to their participants. There were eight repetitive blocks of trials, during which participants performed repeated trials of one of four tasks (addition, subtraction, multiplication, and division). There were four alternating blocks in which participants switched between two tasks: two addition and subtraction blocks and two multiplication and division blocks. On half of all blocks, mathematical symbols reminded participants which mathematical procedure needed to be enacted. On the remaining blocks, no cues were presented. Thus, the type of task (switching or repetitive), the complexity of the tasks (high or low), and the availability of cues were manipulated. Task alternation yielded switching-time costs that increased with rule complexity but decreased with task cuing. It was argued that task switching (goal shifting) is facilitated when explicit cues are made available to indicate the nature of the next task.

We used a computerized version of the above paradigm to permit a more fine-grained analysis of performance than was possible in Rubinstein et al. (2001). There were various reasons for using this paradigm. First, it is an established paradigm for investigating task switching. Second, it permitted investigation of the effects of individual differences in anxiety on several relevant factors, including task switching, task complexity, and cuing (present vs. absent); Rubinstein et al. did not consider individual differences at all. Third, since we were interested in the effects of anxiety on working memory functioning, it was important to include tasks that would involve considerable use of the resources of the working memory system. This is not the case with many of the other task-switching paradigms.

Our central aim was to test one of the main assumptions of ACT: that state anxiety impairs the shifting function. We predicted that state anxiety would impair task switching, and this effect would be greater in high-complexity than in low-complexity tasks, because overall demands on attentional control are higher in high- than in lowcomplexity tasks. We also predicted that the effects of state anxiety would be greater in the absence of cues than in their presence.

\section{METHOD}

\section{Participants}

Fifty-nine undergraduate students at Royal Holloway University of London volunteered for the experiment (further information about the high- and low-anxiety groups is in the Results section). Before the experiment, participants completed the trait form of the StateTrait Anxiety Inventory (STAI-TA; Spielberger, Gorsuch, Lushene, Vagg, \& Jacobs, 1983). The short form of the STAI state scale (Marteau \& Bekker, 1992) was used to measure state anxiety.

\section{Experimental Conditions}

Rule-complexity conditions. As with Rubenstein et al. (2001), there were two rule-complexity conditions: The low-complexity one required solving addition and subtraction problems; the highcomplexity one required solving multiplication and division problems. In keeping with Rubenstein et al., multiplication and division were deemed to have greater complexity because methods of multiplication and division often involve the rules of addition and subtraction as well as other rules. Participants were exposed to blocks of high-complexity trials $50 \%$ of the time and low-complexity blocks the remaining $50 \%$.

Task-cuing conditions. For the cues-present condition, an arithmetic operation sign $(+,-, \times$, or $\div)$ was inserted between the left two-digit and right one-digit numbers, which appeared on the screen on each trial (e.g., $56+7)$. The operation sign indicated the problem type. For the cues-absent condition, there were no operation signs, and participants had to remember their task based on the instructions presented at the start of each block. On half of the trials, the cue was present; on half, it was absent.

Repetitive versus switching conditions. In eight experimental blocks, participants repeated the same mathematical task. In the remaining four blocks, participants were required to alternate (switch) between mathematical tasks. Before commencing uncued switching tasks, participants were given explicit instructions that informed them of the tasks they were to switch between, and which task to begin with.

\section{Apparatus and Design}

The task was designed and programmed using the experimental programming language Presentation Control Language (PCL; www.neurobs.com). There were 12 experimental blocks: 2 blocks of each of the 6 trial types (addition, subtraction, alternating addition/ subtraction, multiplication, division, and alternating multiplication/ division). There were 8 repetitive ( 2 each of addition, subtraction, multiplication, division) and 4 task-switching blocks ( 2 alternating between addition and subtraction, and 2 alternating between multiplication and division; cf. Rubenstein et al., 2001). Cuing was present on half the blocks, absent on the remaining half. The serial order of the different block types was counterbalanced in accordance with Rubenstein et al. (2001).

The stimuli consisted of 144 mathematical problems (12 blocks of 12 trials). In the low-complexity conditions, the two-digit numbers ranged from 12 to 68 and were never integral multiples of 10 . In the high-complexity conditions, the two-digit numbers ranged from 36 to 98 and were never integral multiples of 10 . The one-digit numbers for both conditions ranged from 2 to 9 . Instructions in every block informed participants of the type of mathematical problem to be completed, and whether or not cues would be provided. Each trial began with a two-digit number presented to the left of the center of the computer screen and a one-digit number presented to the right of the center, appearing simultaneously with the cue on cued trials. The stimuli remained on the screen until participants responded by pressing the response button while simultaneously stating aloud the solution to the problem. The answer was recorded by the experimenter. Intertrial interval was $400 \mathrm{msec}$. Speed and accuracy were emphasized. Reaction times (RT) and errors were recorded and saved by the experimental software. 


\section{Procedure}

Participants completed the STAI-TA upon arrival in the laboratory. Instructions on the computer screen told participants they would be asked to solve a series of mathematical problems; they were asked to respond to each problem as quickly and accurately as possible. Following the instructions, participants completed the short form of the STAI state, which was also administered midway and at the end of the experiment. Participants completed some practice trials that preceded the main experiment. The entire session lasted 30-45 min. The experimenter remained in the room, seated behind the participant throughout the session.

\section{RESULTS}

\section{State Anxiety}

On the basis of state anxiety scores at the beginning of the session, individuals were divided into low- $(\leq 13)$ and high- $(\geq 16)$ anxious groups. There were 24 low-anxious $(M=19.71, S D=5.1)$ and 23 high-anxious participants $(M=18.74, S D=1.7)$.

A $2 \times 3$ mixed ANOVA with group (low-anxious, highanxious) as between-subjects factor and time (before, during, end) as within-subjects factor revealed a significant main effect of time $[F(2,90)=26.62, p<.001]$, which was qualified by a linear $[F(1,45)=14.75, p<.001]$ and a quadratic trend $[F(1,45)=42.78, p<.001]$, indicating that anxiety increased from beginning $(M=14.55, S D=3.98)$ to midway $(M=17.8, S D=3.57)$ and decreased toward the end $(M=16.4, S D=3.71)$. The interaction of time $\times$ group $[F(2,90)=8.97, p<.001]$ showed that this pattern was greater in the low-anxious ( $M=11.12$ vs. 16.08 vs. 14.41$)$ than in the high-anxious ( $M=18.13$ vs. 19.60 vs. 18.47$)$ group. Importantly, independent $t$ tests at each time point suggested that high-anxious individuals had higher anxiety across the experiment (all $p \mathrm{~s}<.01$, Bonferroni corrected).

\section{RT Data}

Table 1 presents RTs, error rates, and switching-time cost as a function of trial-block type, rule complexity, and task cuing for each type of arithmetic problem, for the low- and high-anxious groups, respectively.
A $2 \times 2 \times 2 \times 2$ mixed ANOVA was performed with group (low-anxious, high-anxious) as the between-subjects factor and level of complexity (low, high), cuing (present, absent), and task type (repetitive, switching) as withinsubjects factors. As with Rubinstein et al. (2001), there was a significant main effect of complexity $[F(1,45)=$ $114.20, p<.001]$ with faster RTs on low-complexity problems $(M=3,625.80 \mathrm{msec}, S D=1,172.46)$ than on high-complexity problems $(M=7,985.20 \mathrm{msec}, S D=$ $3,420.2)$.

There was a nonsignificant trend for a main effect of task type $[F(1,45)=3.32, p=.07]$, with participants responding faster on repetitive tasks $(M=5,678.83 \mathrm{msec}, S D=$ 2,137.2) than on switching tasks $(M=5,932.26 \mathrm{msec}$, $S D=2,267.17)$. Participants responded slightly faster in the presence $(M=5,788.47 \mathrm{msec}, S D=2,147.51)$ than in the absence $(M=5,822.62 \mathrm{msec}, S D=2,218.80)$ of cues, but this was not significant $[F(1,45)<1$, n.s.; cf. Rubenstein et al., 2001]. The main effect of group was not significant $[F(1,45)=1.29, p=.27]$.

There was a significant interaction of cuing $\times$ task type $[F(1,45)=41.86, p<.001]$, which was qualified by a three-way interaction of cuing $\times$ task type $\times$ complexity $[F(1,45)=39.47, p<.001]$. The interaction of cuing $\times$ task type was considered for each of the low- and high-complexity tasks separately. No significant effects emerged on low-complexity tasks; participants performed similarly on uncued (repetitive, $M=3,541.79 \mathrm{msec}, S D=$ 1,158.04; switching, $M=3,677.42 \mathrm{msec}, S D=1,660.64$ ) and cued (repetitive, $M=3,573.82 \mathrm{msec}, S D=1,166.16$; switching, $M=3,713.68 \mathrm{msec}, S D=1,173.49)$ tasks. However, on high-complexity tasks a significant interaction between cuing and task type was found $[F(1,45)=$ $41.92, p<.001]$. Although cuing facilitated performance on switching tasks (cued, $M=7,145.48 \mathrm{msec}, S D=$ 3,097.28; uncued, $M=9,195.62 \mathrm{msec}, S D=4,609.23$ ) $[t(46)=4.76, p<.001]$, it had the opposite effect on repetitive tasks (cued, $M=8,857.50 \mathrm{msec}, S D=4,545.94$; uncued, $M=6,742.20 \mathrm{msec}, S D=2,522.32)[t(46)=$ $5.66, p<.001]$.

Table 1

\begin{tabular}{|c|c|c|c|c|c|c|c|}
\hline Task Type & $\begin{array}{c}\text { Rule } \\
\text { Complexity }\end{array}$ & Cuing & $\begin{array}{c}\text { Arithmetic } \\
\text { Problem Type }\end{array}$ & $\begin{array}{c}\text { Mean RT } \\
\text { LA }\end{array}$ & $\begin{array}{l}\% \text { Error } \\
\text { LA }\end{array}$ & $\begin{array}{c}\text { Mean RT } \\
\text { HA }\end{array}$ & $\begin{array}{c}\% \text { Error } \\
\text { HA }\end{array}$ \\
\hline \multirow[t]{8}{*}{ Repetitive } & Low & Present & Addition & 3,207 & 4.51 & 3,260 & 5.79 \\
\hline & Low & Present & Subtraction & 3,826 & 7.29 & 4,006 & 2.89 \\
\hline & Low & Absent & Addition & 2,996 & 7.64 & 3,230 & 5.79 \\
\hline & Low & Absent & Subtraction & 3,740 & 8.68 & 4,215 & 8.69 \\
\hline & High & Present & Multiplication & 11,912 & 16.67 & 11,818 & 20.28 \\
\hline & High & Present & Division & 5,438 & 7.99 & 6,276 & 11.59 \\
\hline & High & Absent & Multiplication & 8,807 & 11.80 & 9,311 & 17.02 \\
\hline & High & Absent & Division & 4,207 & 5.56 & 4,607 & 9.42 \\
\hline \multirow[t]{4}{*}{ Switching } & Low & Present & Add/sub & 3,593 & 4.51 & 3,839 & 5.07 \\
\hline & Low & Absent & Add/sub & 3,404 & 6.60 & 3,956 & 6.15 \\
\hline & High & Present & Mult/div & 6,803 & 13.89 & 7,503 & 11.60 \\
\hline & High & Absent & Mult/div & 7,868 & 18.40 & 10,580 & 19.00 \\
\hline
\end{tabular}

Note-HA, high-anxious; LA, low-anxious. 
There was an interaction between cuing and state anxiety $[F(1,45)=7.77, p=.008]$. The high-anxious showed a nonsignificant trend to speed up in the presence of cues $(M=6,006.10 \mathrm{msec}, S D=2,259.46)$ as compared with in their absence $(M=6,305.02 \mathrm{msec}, S D=2,475.81)$ $[t(22)=1.86, p=.07]$, but the low-anxious performed worse in the presence of cues $(M=5,646.79 \mathrm{msec}, S D=$ $2,213.07)$ than in their absence $(M=5,293.43 \mathrm{msec}$, $S D=1,684.70)[t(23)=2.08, p=.049]$. This was evident on repetitive tasks (uncued, $M=49,509.6$; cued, $M=60,957.8$ ), but not on switching tasks (uncued, $M=$ 56,359.1; cued, $M=51,978.0$ ), where cuing facilitated performance.

Of theoretical importance was the significant interaction between state anxiety and task type $[F(1,45)=6.58$, $p=.01]$. Low-anxious participants showed similar performance on switch $(M=5,416.85 \mathrm{msec}, S D=1,702.99)$ and repetitive $(M=5,523.37 \mathrm{msec}, S D=2,197.05)$ $[t(23)<1$, n.s. $]$ trials. In contrast, high-anxious participants responded significantly more rapidly on repetitive ( $M=5,841.05 \mathrm{msec}, S D=2,109.62)$ compared to switch trials $(M=6,470.08 \mathrm{msec}, S D=2,668.9)[t(22)=2.68$, $p=.01]$. This result was precisely in line with the prediction from attentional control theory.

There was a three-way interaction of complexity, task type, and state anxiety $[F(1,45)=4.11, p=.04]$. Therefore, the two-way interaction of task type and anxiety was investigated for the low- and high-complexity tasks separately (Figure 1). For low-complexity tasks, no main effect or interaction reached significance. For high-complexity tasks, analysis revealed a significant interaction of task type $\times$ anxiety $[F(1,45)=4.91, p<.04]$. Whereas lowanxious individuals showed similar performance on repetitive $(M=7,604.65 \mathrm{msec}, S D=3,848.41)$ and switch

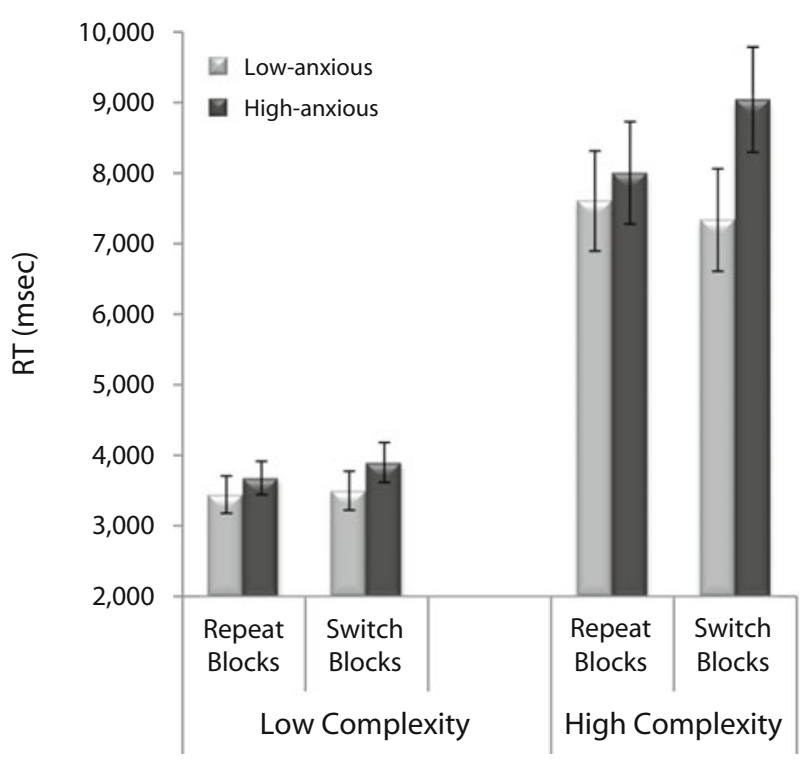

Figure 1. Effects of state anxiety and task switching in each of the low- and high-complexity conditions (bars indicate standard error).
$(M=7,335.46 \mathrm{msec}, S D=3,003.65)$ tasks $[t(23)<1$, n.s.], the high-anxious were slower on switch $(M=$ $9,041.94 \mathrm{msec}, S D=4,088.19)$ compared to repetitive tasks $(M=8,003.54 \mathrm{msec}, S D=3,043.32)[t(22)=2.49$, $p=.02]$. These findings show that task complexity, which influences the demands on attentional control, is an important factor in determining the effects of anxiety on task switching.

\section{Error Rates}

The average error rate across conditions for the lowanxious group was $9.46 \%$ and for the high-anxious group was $10.29 \%[t(45)<1$, n.s.]. There was a weak relationship between error rate and RT ( $r=.29, p=.042)$, indicating that there was no general problem with speedaccuracy trade-off.

\section{Switch Cost}

In keeping with the method employed by Allport, Styles, and Hsieh (1994) and Rubenstein et al. (2001), switch costs $\left(T_{\mathrm{s}}\right)$ were calculated as follows:

$$
T_{\mathrm{s}}=\left[T_{12}-.5\left(T_{1}+T_{2}\right)\right] /(n-1) .
$$

$T_{1}$ and $T_{2}$ are the overall mean RTs for repetitive blocks of trials for the high- and low-complexity tasks, respectively; $T_{12}$ is the overall mean RT for alternating task blocks; and $n-1$ is the number of task switches in an alternating task block of $n$ trials.

A $2 \times 2 \times 2$ mixed ANOVA with complexity and cuing as within-subjects factors and group as between-subjects factor found a main effect of group $[F(1,45)=6.58, p=$ .014]. Low-anxious participants demonstrated a small switch gain overall $(-9.68 \mathrm{msec})$, and high-anxious participants demonstrated a switch cost $(57.17 \mathrm{msec})$. There was a main effect of cuing $[F(1,45)=41.89, p<.0005]$. In the presence of cues, participants demonstrated a switch gain $(-71.24 \mathrm{msec})$, but in the absence of cues they demonstrated a switch cost (118.74 msec).

There was a significant interaction between task complexity and state anxiety $[F(1,45)=4.11, p=.048]$. On low-complexity tasks, both groups equally demonstrated a small switch cost (low-anxious, $5.11 \mathrm{msec}$; high-anxious, $19.97 \mathrm{msec})[t(45)<1$, n.s. $]$. On highcomplexity tasks, the low-anxious group demonstrated a switch gain (low-anxious, $-24.47 \mathrm{msec}$ ), whereas the high-anxious group demonstrated a substantial switch cost $(94.40 \mathrm{msec})[t(45)=2.55, p=.014]$.

\section{DISCUSSION}

According to ACT (Eysenck et al., 2007), anxiety impairs two major functions of the central executive: negative attentional control (inhibition function) and positive attentional control (shifting function). There is much previous empirical support for the predicted adverse effects of anxiety on negative attentional control (see Derakshan et al., 2009). However, with the exception of Ansari et al. (2008), no published studies have considered the predicted effects of anxiety on positive attentional control. Using 
a robust and reliable paradigm developed by Rubinstein et al. (2001) to assess the factors influencing task switching, the present study investigated in an innovative way the effects of state anxiety on the shifting function of working memory, as assessed by task switching. The major findings of this study will be considered within this context.

The single most important theoretical finding was the significant interaction between state anxiety and task type (switching vs. nonswitching). Since task switching involves the shifting function (Miyake et al., 2000), it was predicted from attentional control theory that the demands of task switching would impair the performance of highanxious participants more than that of low-anxious participants. As predicted, task switching had a nonsignificant effect on low-anxious participants but significantly slowed the performance of high-anxious participants. Note that the same tasks were used in the switching and nonswitching conditions, so the intrinsic task demands were equated. Thus, the major difference between the switching and nonswitching conditions was presumably in terms of demands on the shifting function, although other factors may also have been involved (Monsell, 2003).

The further significant interaction between anxiety, task type, and complexity specified more closely the effects of anxiety on task switching. Specifically, this three-way interaction indicated that the adverse effects of anxiety on task-switching performance centered on high-complexity tasks. This is an important finding, because it indicates that anxiety affects task-switching performance only under certain conditions. It is a matter for further research to clarify the appropriate interpretation of this finding. However, the high-complexity tasks probably imposed greater demands on attentional control than the low-complexity tasks did (see Derakshan \& Eysenck, 2009). As a consequence, high-anxious individuals may have found it harder to exert positive attentional control with the high-complexity tasks than with the low-complexity ones.

It is important to note, however, that task complexity was not manipulated to induce different levels of anxiety in low- and high-anxious participants. Our results indicated that task complexity influenced switching performance as a function of participants' state anxiety level at the outset of the experimental trials, with high-anxious participants showing a switch cost. No direct manipulation of complexity, in the context of the present investigation, was intended on anxiety levels. Although low-anxious individuals showed a marked increase in reported anxiety from beginning to midway through the experiment, highanxious individuals reported consistently higher anxiety than did the low-anxious, at all time points. As high- and low-complexity tasks were interleaved throughout the experiment, it was not possible to determine the precise effects of task complexity on state anxiety. Future research should explore the interaction between task complexity and state anxiety systematically by ensuring that the effects of complexity and anxiety are unconfounded.

The third theoretically important finding was the interaction between anxiety and cuing. According to ACT, the impaired shifting function of high-anxious individuals would mean they would be more reliant than low- anxious individuals on explicit cues to facilitate attentional control, and that as a consequence there would be a significant interaction between anxiety and cuing. As predicted, high-anxious individuals performed slower in the absence of cues than in their presence, but the difference just missed significance. The low-anxious individuals responded significantly faster in the absence of cues, indicating that explicit cues had an adverse effect on their performance. A closer look at the data, however, showed that cues facilitated their performance on switching tasks, as expected, but hindered their performance on repetitive trials. It is possible that, in the absence of cuing, the efficiency of the shifting function in low-anxious individuals may have been sufficient for the presentation of explicit cues to distract rather than assist, when tasks were of a repetitive nature.

Several implications should be considered. First, the present findings encourage further research into the effects of anxiety on the shifting function. In particular, the finding that anxiety interacted with shifting with highcomplexity but not with low-complexity tasks deserves to be explored in more detail, across a range of low- and high-complexity tasks.

Second, task-switching paradigms are somewhat complex, in that there are various differences between the task-switching and nonswitching conditions (see Monsell, 2003). Accordingly, it will be important in future to specify more precisely the processes associated with task switching that are affected by anxiety.

Third, in the present study, performance was predicted by state anxiety at the outset of the experiment but not by trait anxiety alone or in conjunction with state anxiety. Clarification of the respective roles of state and trait anxiety could be achieved using a design in which groups low and high in trait anxiety were exposed to low- and high-stress conditions designed to manipulate state anxiety levels.

Fourth, although Miyake et al. (2000) identified three major functions of the central executive, it is entirely possible that additional functions should be identified. For example, Collette and van der Linden (2002) identified what is potentially an important fourth function: dual-task coordination. It is of theoretical interest to see whether dual-task coordination involves attentional control. If it does, it would then be predicted from ACT that anxiety should impair performance, if such coordination were required.

In sum, the central finding that anxiety impaired the shifting function supports a major assumption of ACT that has very rarely been tested. In addition, we have identified some of the factors determining when anxiety impairs the shifting function and when it does not. This provides a solid foundation for future research. It is important for such research to consider paradigms other than the one we used in order to assess the generality of our findings.

\section{AUTHOR NOTE}

The research reported here was supported by an ESRC grant awarded to N.D. and M.W.E. The authors are grateful for the council's generous support. Correspondence concerning this article should be addressed 
to N. Derakshan, Affective and Cognitive Neuroscience Lab, School of Psychology, Birkbeck University of London, Malet Street, London WC1E 7HX, England (e-mail: n.derakhshan@bbk.ac.uk).

\section{REFERENCES}

Allport, A., Styles, E., \& Hsieh, S. (1994). Shifting intentional set: Exploring the dynamic control of tasks. In C. Umiltà \& M. Moscovitch (Eds.), Attention and performance XV: Conscious and nonconscious information processing (pp. 421-452). Cambridge, MA: MIT Press.

ANSARI, T. L., DeraKshan, N., \& Richards, A. (2008). Effects of anxiety on task switching: Evidence from the mixed antisaccade task. Cognitive, Affective, \& Behavioral Neuroscience, 8, 229-238.

BAdDELEY, A. D. (1986). Working memory. Oxford: Oxford University Press, Clarendon Press.

Collette, F., \& VAn der Linden, M. (2002). Brain imaging of the central executive component of working memory. Neuroscience \& Biobehavioral Reviews, 26, 105-125.

Derakshan, N., Ansari, T. L., Hansard, M., Shoker, L., \& Eysenck, M. W. (2009). Anxiety, inhibition, efficiency, and effectiveness: An investigation using the antisaccade task. Experimental Psychology, 56, 48-55.

Derakshan, N., \& Eysenck, M. W. (2009). Anxiety, processing efficiency, and cognitive performance: New developments from attentional control theory. European Psychologist, 14, 168-176.

EysencK, M. W., \& Calvo, M. G. (1992). Anxiety and performance: The processing efficiency theory. Cognition \& Emotion, 6, 409-434.
Eysenck, M. W., Derakshan, N., Santos, R., \& Calvo, M. G. (2007). Anxiety and cognitive performance: Attentional control theory. Emotion, 7, 336-353.

Eysenck, M. W., Payne, S., \& Derakshan, N. (2005). Trait anxiety, visuospatial processing, and working memory. Cognition \& Emotion, 19, 1214-1228.

Friedman, N. P., \& MiyaKe, A. (2004). The relations among inhibition and interference control functions: A latent-variable analysis. Journal of Experimental Psychology: General, 133, 101-135.

Marteau, T. M., \& BeKKer, H. (1992). The development of a six-item short-form of the state scale of the Spielberger State-Trait Anxiety Inventory (STAI). British Journal of Clinical Psychology, 31, 301-306.

Miyake, A., Friedman, N. P., Emerson, M. J., Witzki, A. H., HowERTER, A., \& WAGER, T. D. (2000). The unity and diversity of executive functions and their contributions to complex "frontal lobe" tasks: A latent variable analysis. Cognitive Psychology, 41, 49-100.

Monsell, S. (2003). Task switching. Trends in Cognitive Sciences, 7 , 134-140.

Rubinstein, J. S., Meyer, D. E., \& Evans, J. E. (2001). Executive control of cognitive processes in task switching. Journal of Experimental Psychology: Human Perception \& Performance, 27, 763-797.

Spielberger, C. D., Gorsuch, R. L., Lushene, R., Vagg, P. R., \& JACOBS, G. A. (1983). Manual for the State-Trait Anxiety Inventory. Palo Alto, CA: Consulting Psychologists Press.

(Manuscript received February 2, 2009; revision accepted for publication July 14, 2009.) 